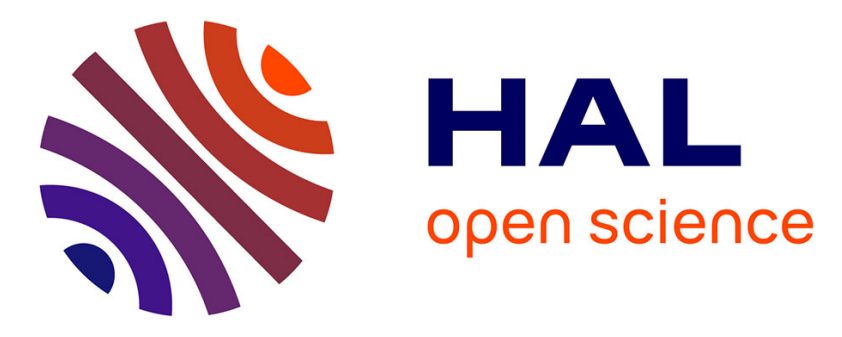

\title{
Scintillation Signal in XEMIS2, a Liquid Xenon Compton Camera with $3 \gamma$ Imaging Technique
}

Yuwei Zhu, M. Abaline, S. Acounis, N. Beaupère, J.L. Beney, J. Bert, S. Bouvier, P. Briend, J. Butterworth, T. Carlier, et al.

\section{To cite this version:}

Yuwei Zhu, M. Abaline, S. Acounis, N. Beaupère, J.L. Beney, et al.. Scintillation Signal in XEMIS2, a Liquid Xenon Compton Camera with $3 \gamma$ Imaging Technique. 4th International conference on Technology and Instrumentation in Particle Physics, May 2017, Beijing, China. pp.159-163, 10.1007/978981-13-1316-5_30. hal-01863099

\section{HAL Id: hal-01863099 \\ https://hal.science/hal-01863099}

Submitted on 25 Nov 2021

HAL is a multi-disciplinary open access archive for the deposit and dissemination of scientific research documents, whether they are published or not. The documents may come from teaching and research institutions in France or abroad, or from public or private research centers.
L'archive ouverte pluridisciplinaire HAL, est destinée au dépôt et à la diffusion de documents scientifiques de niveau recherche, publiés ou non, émanant des établissements d'enseignement et de recherche français ou étrangers, des laboratoires publics ou privés. 


\title{
Scintillation Signal in XEMIS2, a Liquid Xenon Compton Camera with $3 \gamma$ Imaging Technique
}

Y. Zhu ${ }^{1(\bowtie)}$, M. Abaline ${ }^{2}$, S. Acounis ${ }^{1}$, N. Beaupère ${ }^{1}$, J. L. Beney ${ }^{1}$, J. Bert ${ }^{3}$, S. Bouvier ${ }^{1}$, P. Briend ${ }^{4}$, J. Butterworth ${ }^{4}$, T. Carlier ${ }^{5}$, H. Chanal ${ }^{2}$, M. Cherel $^{6}$, J. P. Cussonneau ${ }^{1}$, M. Dahoumane ${ }^{7}$,

L. Gallego-Manzano ${ }^{1}$, D. Giovagnoli ${ }^{3}$, J. Idier $^{8}$, F. Kraeber-Bodere ${ }^{5}$, P. Le Ray ${ }^{1}$, F. Lefèvre ${ }^{1}$, O. Lemaire ${ }^{1}$, S. Manen ${ }^{2}$, J. Masbou ${ }^{1}$, H. Mathez ${ }^{7}$, E. Morteau ${ }^{1}$, N. Pillet ${ }^{2}$, D. Roy ${ }^{1}$, L. Royer ${ }^{2}$, M. Staempflin ${ }^{4}$, J. S. Stutzmann ${ }^{1}$, R. Vandaele ${ }^{2}$, L. Virone ${ }^{1}$, D. Visvikis ${ }^{3}$, Y. Xing ${ }^{1}$, and D. Thers ${ }^{1}$

1 SUBATECH, IMT Atlantique, CNRS/IN2P3, Université de Nantes, 44307 Nantes, France

Yuwei.Zhu@subatech. in2p3.fr

${ }^{2}$ LPC Clermont-Ferrand, 24 Avenue des Landais, Clermont-Ferrand, France

3 INSERM, UMR1101, LaTIM, CHRU Morvan,

2 Avenue Foch, 29600 Brest, France

4 AIR LIQUIDE Advanced Technologies Division, 38360 Sassenage, France

${ }^{5}$ Centre Hospitalier Universitaire de Nantes,

1 place Alexis-Ricordeau, 44093 Nantes, France

${ }^{6}$ INSERM U892 équipe 13, 8 quai Moncousu, 44000 Nantes, France

7 IPNL Université de Lyon, CNRS/IN2P3 UMR5822, Lyon, France

8 LS2N, Ecole Centrale de Nantes, CNRS/Inp, Université de Nantes, 44307 Nantes, France

\begin{abstract}
The XEMIS project (XEnon Medical Imaging System), which makes use of $3 \gamma$ imaging technique and liquid xenon Compton camera, aims to make a precise $3 \mathrm{D}$ localization of a specific radioactive emitter and to reduce drastically (100 times less) the injected activity to the patient in cancer diagnosis. The $3 \gamma$ imaging is characterized by the simultaneous detection of $3 \gamma$-rays emitted by ${ }^{44} \mathrm{Sc}$ which is a $(\beta+, \gamma)$ emitter. The second prototype XEMIS2 is a liquid xenon cylindrical camera for small animal imaging. The active volume of XEMIS2 is surrounded by a set of VUV-sensitive Hamamatsu photomultipliers, for the scintillation signals detection. A pulse-shaping amplifier was tested in XEMIS1 for the readout of the scintillation signal of the PMT. The typical output pulse shows a relatively good performance of the pulse-shaping amplifier providing a possible solution for XEMIS2 scintillation DAQ. Meanwhile, the pulse-shaping amplifier and the constant fraction discriminator (CFD) have lay the foundation of the preliminary design of XEMIS2 scintillation signal detection chain.
\end{abstract}

Keywords: Medical imaging $\cdot 3 \gamma$ imaging $\cdot$ Scintillation signal Liquid xenon 


\section{Introduction}

An innovative liquid xenon Compton camera project, XEMIS (XEnon Medical Imaging System), based on the $3 \gamma$ imaging technique [1], has been proposed by Subatech laboratory. This project consists of 3 main steps: XEMIS1, a small dimension prototype of a single-phase liquid xenon Compton camera, has been successfully developed providing the experimental feasibility of $3 \gamma$ imaging technique. XEMIS2 which is a larger scale liquid xenon cylindrical camera for small animal imaging has been designed for preclinical application in hospital center, and is now under qualification. The long-term objective, XEMIS3, which is a larger liquid xenon Compton camera, will be oriented to whole human body imaging application.

\section{XEMIS2: Small Animal Imaging}

The XEMIS2 is based on the measurement of both scintillation and ionization signals, generated after the interaction of an $\gamma$-ray with LXe. XEMIS2 consists of three main parts: the camera, the purification system and the cryogenic storage and recovery system called ReStoX. The XEMIS2 camera contains two LXe time projection chamber (TPC), $12 \mathrm{~cm}$ drift length each (Fig. 1). When a $\gamma$-ray interacts with LXe, due to the fast scintillation light emission, the arrival time of the VUV scintillation photons to the PMT provides the interaction time $t_{0}$. The electric field forces the ionized electrons to drift towards the segmented anode and finally forms the ionization signals, that permits us to get the position and deposited energy of each interaction vertex. High drift field is needed to reduce the recombination process for good ionization signal measurement. Meanwhile, it reduces the VUV light production. At XEMIS2 drift field of $2 \mathrm{kV} / \mathrm{cm}$, the scintillation yield is around 20000 photons $/ \mathrm{MeV}$ [2].

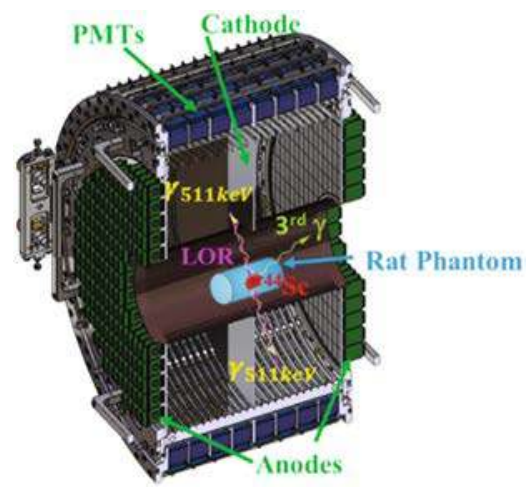

Fig. 1. The active zone of the XEMIS2 prototype

There are two anodes on both sides and a cathode in the center of XEMIS2 camera. Each anode is segmented in 10000 pixels with ultra-low noise ionization read out. 
To detect the scintillation signals, the active volume of XEMIS2 is surrounded by 64 PMTs covering 16 sectors in $\varphi$, in a preliminary stage. For the future upgrade, the scintillation light will be detected by 380 PMTs which can make a complete coverage of the active zone. There is no added global trigger system for physic event selection in XEMIS2, the self-triggered PMT channels will be utilized. To reduce the data flow from DAQ, the pulse from the PMT needs to be first treated with a detection chain to obtain the interaction time and the charge of each scintillation signal.

\section{Scintillation Signal Detection System of XEMIS}

\subsection{The Photomultiplier Tube (PMT)}

To detect the scintillation photons with wavelengths of $178 \mathrm{~nm}$ in XEMIS2, the VUVsensitive Hamamatsu R7600-06MOD-ASSY PMT which spectral sensitivity is from 160 to $650 \mathrm{~nm}$ was selected. The PMT has a quartz synthetic silica window and a bialkali photocathode with a minimum active area of $18 \times 18 \mathrm{~mm}^{2}$. This type of PMT is able to work directly at $-110{ }^{\circ} \mathrm{C}$ at a pressure of 1 bar in the LXe, which shows a good UV photon sensitivity with quantum efficiency of $35 \%$ at $178 \mathrm{~nm} \mathrm{[3].}$

\subsection{The Pulse-Shaping Amplifier}

In order to filter and shape the output pulse of the PMT in XEMIS2, we developed a pulse-shaping amplifier. It consists of a sixth order RLC low-pass filter which was inspired by the work of ATLAS Tile Calorimeter [4]. By considering the effects of different electrical components, PSpice software was used to simulate the output shaped pulses. We also considered the non-perfect inductance with the effects of conductance and resistance in the simulation. The peaking time of the shaped pulse which depends on different values of electrical components affects the pulse height. The pulse-shaping amplifier attenuates the high frequency noise components. The height of the output shaped pulse is correlated to the number of photoelectrons on the PMT.

\subsection{Experimental Results with XEMIS1}

The pulse-shaping amplifier was tested in XEMIS1 with a sealed gamma sources ${ }^{22} \mathrm{Na}$. The trigger system of XEMIS1 uses the quad coincidence for physic event selection. The duration time of the output pulse generated by PMT is normally shorter than $100 \mathrm{~ns}$. Besides, the typical output pulse generated by the pulse-shaping amplifier has a quasi-gaussian shape with a peaking time about $60 \mathrm{~ns}$ (Fig. 2) which avoids the pile-up phenomenon. Meanwhile, this peaking time is longer than the decay time of LXe (3\% singlet excited state with decay time of $2.2 \mathrm{~ns}, 60 \%$ triplet excited state with $27 \mathrm{~ns}$ and $37 \%$ recombination about $45 \mathrm{~ns}$ for an electric field of $2 \mathrm{kV} / \mathrm{cm}[3,5])$. 

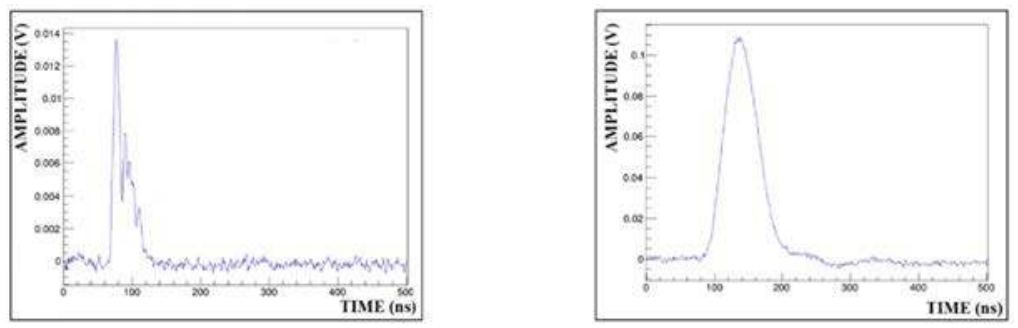

Fig. 2. The examples of output pulses generated by PMT (left) and pulse-shaping amplifier (right) in XEMIS1

\subsection{Preliminary Design of XEMIS2 Scintillation Signal Detection Chain}

A constant fraction discriminator (CFD) is designed to mimic the mathematical operation of finding the maximum of a pulse by finding the zero-crossing point (Fig. 3). With the CFD method, the triggering time is independent of the pulse height which avoids the time walk phenomenon. In addition, the CFD module has a threshold for suppressing noise events.

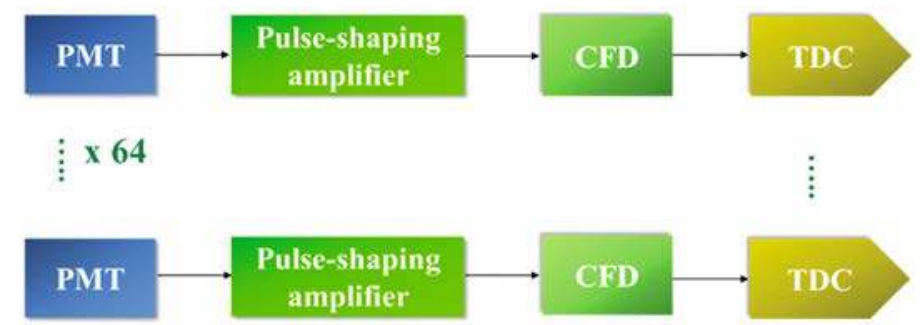

Fig. 3. Preliminary design of XEMIS2 scintillation signal detection chain

\section{Conclusion and Perspective}

The XEMIS1 results showed that the pulse-shaping amplifier has a relatively good performance which provides a possible solution for XEMIS2 scintillation DAQ. In the first place, we can make a precise time measurement by using CFD method applied on the shaped pulse. Besides, by combining PMT signals with the additional information provided by the ionization signal, it is possible to reconstruct the $\mathrm{Z}$ position of each interaction point inside the detector. Furthermore, in order to reduce the occupancy of the TPC, the scintillation signals will be utilized for spatial pre-localization of each $\gamma$ ray interactions allowing to handle a 10-fold higher activity by using 380 PMTs. 


\section{References}

1. Grignon, C., et al.: Nuclear medical imaging using $\beta+\gamma$ coincidences from Sc-44 radionuclide with liquid xenon as detector medium. Nucl. Instrum. Methods A 571(1), 142-145 (2007)

2. Doke, T., et al.: Absolute scintillation yields in liquid argon and xenon for various particles. Jpn. J. Appl. Phys. 41(3R), 1538 (2002)

3. Gallego Manzano, L.: Optimization of a single-phase liquid xenon Compton camera for $3 \gamma$ medical imaging. PhD diss., Nantes, Ecole des Mines (2016)

4. Tang, F., et al.: Design of the front-end readout electronics for ATLAS tile calorimeter at the sLHC. IEEE Trans. Nucl. Sci. 60(2), 1255-1259 (2013)

5. Kubota, S., et al.: Dynamical behavior of free electrons in the recombination process in liquid argon, krypton, and xenon. Phys. Rev. B 20(8), 3486 (1979) 